Mens

Revue d'histoire intellectuelle et culturelle

Yvan Lamonde. Fais ce que dois, advienne que pourra :

Papineau et l'idée de nationalité, Montréal, Lux éditeur, 2015, $240 \mathrm{p}$.

\title{
Julie Guyot
}

Volume 17, numéro 1-2, automne 2016, printemps 2017

URI : https://id.erudit.org/iderudit/1050795ar

DOI : https://doi.org/10.7202/1050795ar

Aller au sommaire du numéro

Éditeur(s)

Centre de recherche en civilisation canadienne-française

ISSN

1492-8647 (imprimé)

1927-9299 (numérique)

Découvrir la revue

Citer ce compte rendu

Guyot, J. (2016). Compte rendu de [Yvan Lamonde. Fais ce que dois, advienne que pourra: Papineau et l'idée de nationalité, Montréal, Lux éditeur, 2015,

240 p.] Mens, 17(1-2), 186-190. https://doi.org/10.7202/1050795ar d'utilisation que vous pouvez consulter en ligne.

https://apropos.erudit.org/fr/usagers/politique-dutilisation/ 
travers. Il y aura un deuxième tome à Celebrating Canada. Espérons qu'il corrigera l'image problématique laissée par le premier.

- Patrice Groulx

Université Laval

\section{Yvan Lamonde. Fais ce que dois, advienne que pourra: Papineau et l'idée de nationalité, Montréal, Lux éditeur, 2015, 240 p.}

Dans une prose limpide et une structure synthétique, l'historien Yvan Lamonde offre, avec cet ouvrage, une excellente entrée en matière à quiconque souhaite découvrir et comprendre la pensée politique de Louis-Joseph Papineau, de même que les choix de ce dernier tout au long de son parcours professionnel. Dans Fais ce dois, advienne que pourra: Papineau et l'idée de nationalité, Lamonde expose le contexte dans lequel la position constitutionnelle du chef du mouvement patriote évoluera au fil des ans sur des questions clés telles que l'émancipation, la séparation et l'indépendance. "Jusqu'où Papineau est-il allé dans la formulation de sa conviction " de leur nécessité pour le Bas-Canada? (p. 10) De manière fort pertinente, l'incidence inéluctable de la conjoncture tant dans la formation d'une pensée politique que dans la mise en place d'une action politique elle-même est constamment réitérée dans cet ouvrage. Ainsi, Yvan Lamonde présente une conception de la nationalité chez Papineau marquée par l'américanité, par un certain type de républicanisme qui en découlera, puis, enfin, par la contingence du colonialisme britannique. En exergue est proposé un bel instantané du personnage qu'était Louis-Joseph Papineau, des principes qui allaient nourrir ses objectifs et sur lesquels son action allait s'appuyer de 1808 à 1871 . En plus de la devise du grand parlementaire qui sert de titre au présent livre, un extrait d'une lettre rédigée en février 1839 et destinée à l'abbé Étienne Chartier constituent ce portrait : « [...] j'ai tâché de le servir [le pays], au degré et par les voies qui me paraissaient possibles [...]. Mon jugement et ma conscience seront toujours mes premiers conseillers. » 
L'analyse s'appuie essentiellement sur trois types de sources : la correspondance expédiée de Papineau, ses interventions publiques (notamment ses discours à la Chambre et lors des assemblées publiques de 1837), puis ses mises en récit de l'expérience bas-canadienne (Histoire de la résistance du Canada au gouvernement anglais, [1839], et Cette fatale Union: adresses, discours et manifestes [1847-1848]). Les versions électroniques de ces documents ont été interrogées à l'aide de 24 mots clés et selon la méthode d'analyse du discours politique développée par les historiens Louis-Georges Harvey et Mark V. Olsen à la fin des années 1980.

L'ouvrage, divisé en sept chapitres, couvre la période 1808-1871. Cinq chapitres font état à proprement dit de l'histoire constitutionnelle bas-canadienne, en présentant de manière efficace le statut et le rôle de Papineau à chacune des étapes présentées. La subdivision chronologique choisie (1808-1823; 1824-décembre 1837 ; décembre $1837-1845$; 1840-1845; 1845-1871) illustre parfaitement à la fois les fameuses « contingences du colonialisme " venues du double front que constituaient Londres et l'administration locale, puis l'évolution du parcours idéologique et politique du chef patriote dans la colonie et en exil. À l'intérieur de cette subdivision, les personnages clés et les rouages du système politique bas-canadien sous le Régime britannique sont bien définis. Les deux derniers chapitres présentent une réflexion sur la notion de nationalité chez Papineau et illustrent la place occupée par la référence états-unienne dans la pensée et l'action de ce dernier.

Le chapitre 1, «La voie de la représentation démocratique (18081823) ", est consacré à la présentation de Papineau. Ses origines familiales, sociales, sa formation et son entrée précoce à la Chambre d'assemblée du Bas-Canada. Lamonde retrace le cadre à l'intérieur duquel se définit le britannisme de celui qui sera l'orateur de la Chambre d'assemblée de façon quasi ininterrompue de 1815 à 1837. La Constitution de 1791 concède des droits aux Canadiens à titre de sujets britanniques et reconnaît une assemblée élective, véritable moyen de résistance des députés libéraux et patriotes contre une 
administration qui favorise " les intérêts des tories de la colonie " (p. 18). Cependant, les écueils que rencontrera la majorité de l'Assemblée, puis l'élaboration à Londres, en 1822, d'un projet d'Union du Haut et du Bas-Canada feront en sorte que, pour Papineau, "les choses ne seront jamais plus les mêmes après 1822 "(p. 21).

À la suite de ses séjours en Angleterre, en France et aux ÉtatsUnis en 1823, Papineau voit ses opinons politiques ancrées de manière de plus en plus décisive du côté américain. C'est l'état de la société qui l'intéresse; il déplore notamment que les différences sociales soient marquées en France et en Angleterre et que l'influence de l'Église se manifeste encore dans la sphère publique (p. 39-43).

Dans le deuxième chapitre, il est question de la nécessité d'obtenir un Conseil législatif « tiré du peuple » (1824-décembre 1837). Papineau, qui verra au cours de sa carrière se succéder huit gouverneurs et qui vivra trois prorogations de la Chambre (sous Dalhousie en mars 1827, sous Aylmer en mars 1834, sous Gosford en mars 1836) en moins de dix ans, a très tôt saisi qu'il fallait mettre fin à la «tyrannie organisée " (p. 50). Pour ce faire, l'une des réformes essentielles consistait à soumettre la sélection des membres du Conseil législatif au choix populaire. Lamonde fait d'ailleurs état de « l'escalade dans les déceptions " de Papineau entre 1823 et 1834, notamment face $\mathrm{au}$ "vice essentiel du régime colonial », c'est-à-dire le patronage, ce qui mènera le chef patriote vers un " positionnement démocratique et républicain» (p. 55, 56, 58, 66).

Yvan Lamonde montre qu’à compter de 1834 , les références aux termes " nationalité " et "nation " seront plus fréquentes dans le discours de Papineau. Ce dernier est déterminé à faire échec à la politique métropolitaine de "diviser pour régner ", qui exacerbe les antipathies nationales. Dans le modèle anticolonial que Papineau veut développer, il s'inspire de la pensée de Thomas Paine - dont sa célèbre formule : "Nulle nation n'en peut commander une autre " (p. 78) - et insiste sur le fait que seule la souveraineté du peuple est source de pouvoir (p. 71). 
Le troisième chapitre, intitulé «Désaccord sur la frontière étatsunienne, indifférence dans la métropole française (décembre 18371845) », porte sur l'exil de Papineau. Il y est question, entre autres, de la correspondance de Papineau avec le célèbre historien George Bancroft, dans laquelle le Bas-Canadien explique à l'Américain le contexte du soulèvement de 1837 et lui demande conseil pour la suite des choses. Lamonde fait également état de la réunion des patriotes exilés en janvier 1838 à Middlebury, de la correspondance "du chef " avec divers personnages clés en France et en Angleterre en vue d'obtenir des appuis pour une éventuelle résistance armée. Le contexte international de la fin des années 1830 n'est pas celui du dernier quart du XvIII ${ }^{\mathrm{e}}$ siècle : il faut préserver la balance du pouvoir politique entre les grandes puissances européennes et les États-Unis, et ainsi un contexte de paix. Les appuis ne viendront pas.

Dans le quatrième chapitre, «L'Union et le gouvernement responsable vus de Paris (1840-1845) ", Lamonde montre l'influence de l'exil de Papineau en France sur sa réflexion à propos de la nationalité. Les questions politiques et constitutionnelles qui animaient le BasCanada d'alors, telles que la disparition de la représentation proportionnelle et l'octroi d'un gouvernement dit " responsable ", marqueront de façon définitive les positions de Papineau. Élément incontournable, l'auteur approfondit ici la notion de responsabilité législative, qu'il avait d'abord exposée dans Papineau : erreur sur la personne (avec Jonathan Livernois, Éditions du Boréal, 2012).

Le chapitre 5, «Face à l'hiver de l'isolement britannique, l'émancipation continentale (1845-1871) ", fait état de la dernière étape de l'engagement politique du grand patriote. Les années 1848 et 1849 sont décisives. En 1848, Papineau, de retour sur la scène parlementaire, dénoncera l'Union et continuera de propager l'idée qu'il faut conjuguer républicanisme et nationalité. Pour ce faire, il est impératif de reconnaître les mêmes droits (civils, religieux, politiques) à « tous les hommes, sans distinction, de leur culte, ni du pays de leur naissance» (p. 139). L’année 1849 sera celle de la marginalisation du grand parlementaire et de sa prise de position pour l'annexion aux États-Unis. 
L'avant-dernier chapitre intitulé " Papineau et l'idée de nationalité " fait la genèse de ce que le célèbre parlementaire bas-canadien « a pu faire » à propos de l'émancipation, de la séparation et de l'indépendance du Bas-Canada. Lamonde rappelle les différentes fractures qui ont, entre 1823 et 1871 , conduit à l'idée d'une "souveraineté possible » à l'intérieur d'un fédéralisme américain (p. 186, 196). Cette posture politique serait « inévitablement un révélateur du type de colonialisme pratiqué par l'Angleterre au XIX ${ }^{e}$ siècle» (p. 194).

Le dernier chapitre, «La référence à Jefferson et la culture étatsunienne de Papineau ", constitue un rappel des moments clés dans l'évolution du " credo politique " du chef patriote vers son adhésion à la pensée républicaine américaine par l'entremise des actions des Washington, Jefferson et Jackson.

Yvan Lamonde souhaitait dissiper le nuage de brume qui recouvre, encore aujourd'hui, la mémoire de Papineau (p. 9). Le pari est gagné. Il a notamment fait deux mises au point fondamentales : il a expliqué et remis en contexte la conception de la nationalité du chef patriote, puis sa définition de la responsabilité législative en régime démocratique. À la lumière de l'historiographie récente, il était primordial de remettre les pendules à l'heure.

- Julie Guyot Département d'histoire Cégep Édouard-Montpetit

\section{Serge Dupuis. Plus peur de l'hiver que du Diable : une his- toire des Canadiens français en Floride, Sudbury, Éditions Prise de parole, 2016, $188 \mathrm{p}$.}

Les différents aspects de la grande migration qui a mené près d'un million de Canadiens français vers le Nord-Est et le Midwest des États-Unis entre 1840 et 1930 sont maintenant bien connus. Les études d'Yves Roby, de Bruno Ramirez, d'Yves Frenette et du duo Éric Waddell et Dean Louder ont, entre autres, permis d'évaluer de manière précise les différentes articulations sociotemporelles de ce large mouvement qui demeure un événement majeur de l'histoire 